\section{Proton beam therapy for iris melanoma: a review of 15 cases}

P Rundle', AD Singh ${ }^{2}$ and I Rennie ${ }^{1}$

\begin{abstract}
Aim To report results of proton beam therapy for iris melanoma.

Methods A retrospective case series of $\mathbf{1 5}$ patients with nonresectable iris melanomas treated with proton beam therapy between August 1998 and August 2004. The main outcome measures were (1) local tumour control, (2) complications, and (3) eye retention.

Results Of the 15 cases, 11 patients showed documented growth (including two cases of local recurrence following iridocyclectomy) while a further three cases were biopsy-proven melanoma. One patient presented with a newly acquired vascular nodule of the iris associated with angle seeding and glaucoma. Tumour control at mean follow-up of 34 months was $93 \%$ (14 of 15 eyes). Common complications included glaucoma in $53 \%$ (five patients had glaucoma prior to irradiation), dry eye $(27 \%)$ and cataract in three patients (20\%). Eye retention was possible in $80 \%$ (12 cases).

Conclusion Proton beam therapy is an effective treatment for cases of nonresectable iris melanoma. The major complications are cataract and glaucoma.

Eye (2007) 21, 79-82. doi:10.1038/sj.eye.6702132; published online 13 January 2006
\end{abstract}

Keywords: iris melanoma; proton beam; uveal

\section{Introduction}

The iris is the least common site for uveal melanoma accounting for only $3 \%$ of the cases. ${ }^{1}$ Furthermore, iris melanomas tend to behave in an indolent fashion and carry a much better prognosis than either ciliary body or choroidal melanomas. ${ }^{2}$ For this reason, the mainstay of treatment for localised iris melanomas is resection by means of an iridectomy or iridocyclectomy. In cases of diffuse melanomas, particularly if the intraocular pressure is raised, enucleation is frequently necessary.

Brachytherapy is also an option in selected cases. Several authors have described the use of proton beam therapy for iris melanomas although no large case series have been published. ${ }^{3,4}$ Over the last 5 years, we have used proton beam therapy in 15 patients with iris melanomas of varying complexity and in this report we present our initial results.

\section{Materials and methods}

Patient details of all ocular oncology patients seen in Sheffield are stored on a dedicated oncology database. A search of these records identified all patients with iris melanomas treated with proton beam therapy. A total of 15 patients were identified. Information regarding age, sex, laterality, Snellen visual acuity, indications for treatment, intraocular pressure, length of follow-up, and complications was collected. All patients were treated with a standard protocol of 5310 cGy delivered in four fractions. No localising markers were required and localisation was based on anterior segment photographs, ultrasound biomicroscopy, axial length, and measurement of corneal diameters. EYEPLAN software was used to formulate an individual treatment plan for each patient (Figure 1a). Immobilisation of the head was achieved by means of a custom-made mask and bite block, while immobilisation of the eye was achieved by means of viewing a fixation light. The eyelids were retracted out of the radiation field. The pupil was dilated to reduce the treatment volume. Patients were reviewed at regular intervals with measurement of intraocular pressure, slit-lamp assessment, anterior segment photography, and ultrasound biomicroscopy as required.
${ }^{1}$ Ocular Oncology Clinic, Royal Hallamshire Hospital, Sheffield, UK

${ }^{2}$ Department of Ophthalmic Oncology, Cole Eye Institute (13-129), Cleveland Clinic Foundation, Cleveland, $\mathrm{OH}$, USA

Correspondence: P Rundle, Department of Ophthalmology, Royal Hallamshire Hospital, Glossop Road, Sheffield S10 2JF, UK

Tel: + 441142713619 ;

Fax: + 441142713682 .

E-mail: Paul.Rundle@

sth.nhs.uk

Received: 29 April 2005 Accepted in revised form: 26 August 2005

Published online: 13 January 2006

The authors have no proprietary interests in the work described 

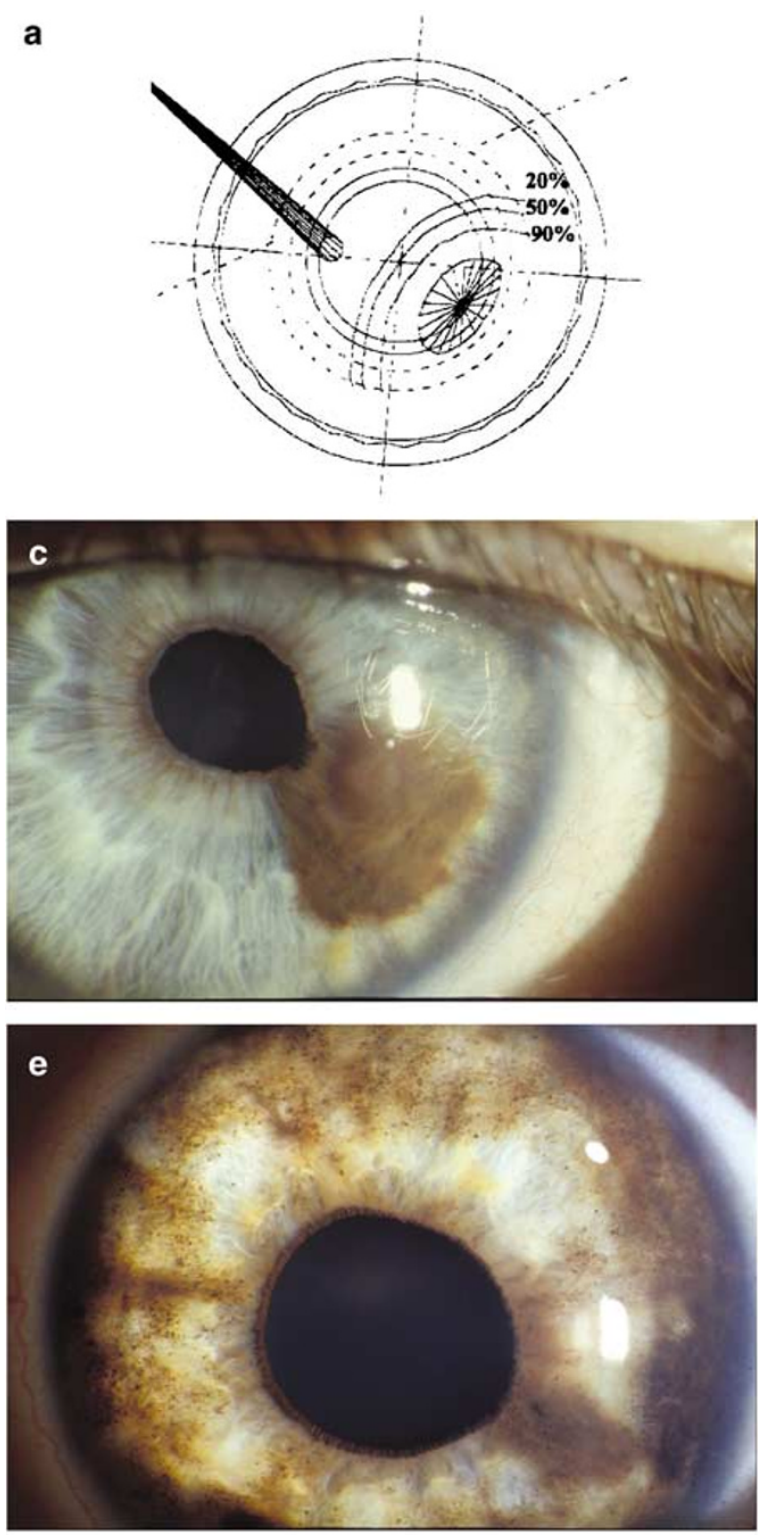
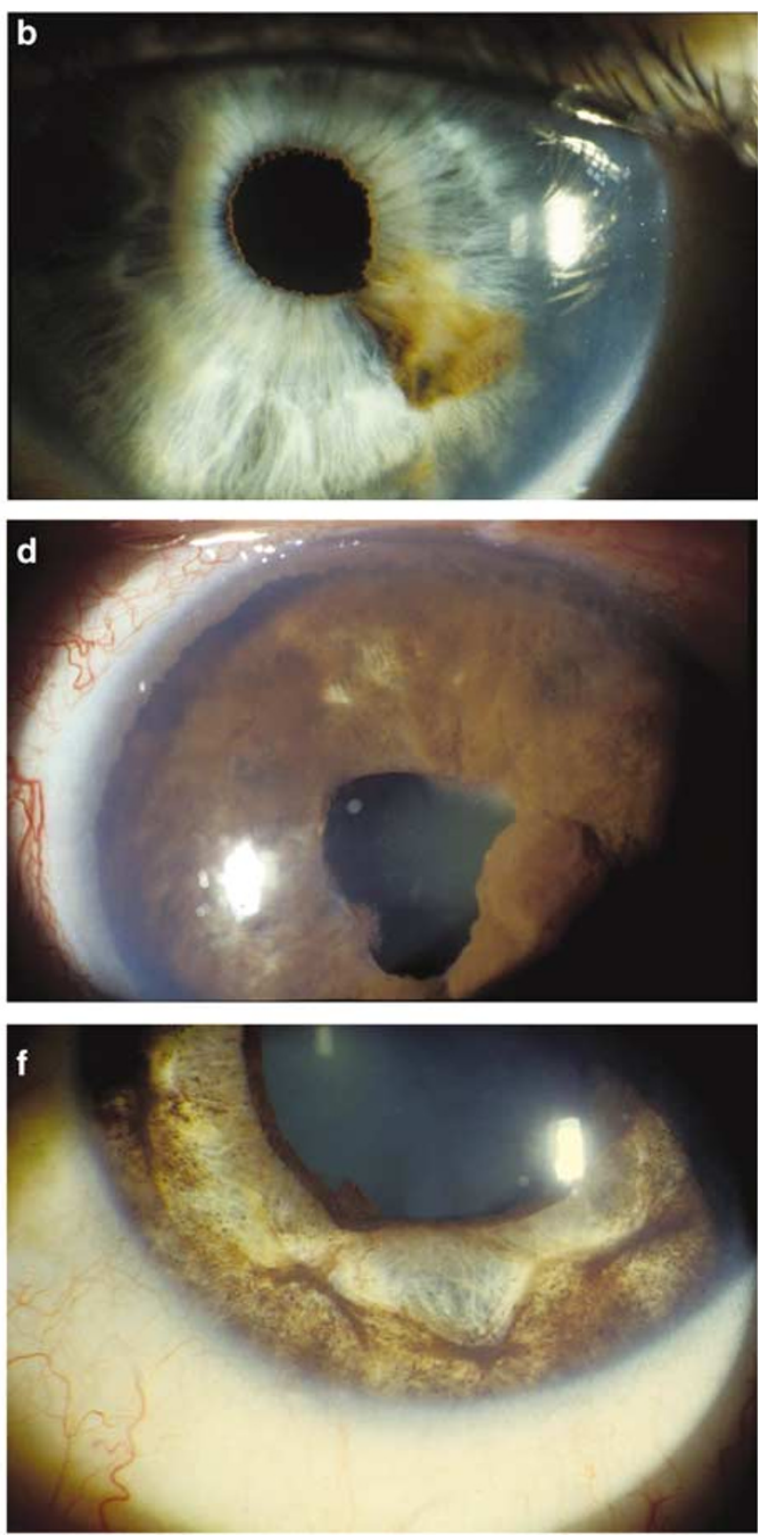

Figure 1 (a) Treatment plan showing 20, 50, and 90\% isodose curves for the patient in (b). (b) A 51-year-old male with documented growth of an iris lesion in his only seeing, left eye; (b) appearance in 1993; (c) appearance in 1998; (d) appearance in 2004 with massive recurrence of the iris melanoma following proton beam therapy. The eye was enucleated. (e) A 30 year old female with diffuse iris melanoma of the left eye at presentation and (f) 2 years post-proton beam therapy. Note the iris atrophy and posterior synechiae.

\section{Results}

In total 15 patients were identified, 12 females and three males. The mean age was 41 years (range 11-61 years). In nine cases, the left eye was involved and in six cases, the right eye was affected. As would be expected there were no bilateral cases. In all, 13 patients had pretreatment Snellen acuity better than $6 / 9$, one had $6 / 12$, and one patient $6 / 36$. Four melanomas were considered focal ( 3 clock hours or less) and 11 were classified as diffuse. This latter group included two cases of recurrence postiridectomy.

Indications for treatment were documented growth in nine cases occurring over periods of follow-up ranging from 4 to 120 months. Four of these patients underwent fine-needle biopsy. A further three patients underwent biopsy of a suspicious lesion at presentation (seven biopsies in total). Two patients had recurrence following iridectomy. One patient presented with a vascular iris nodule associated with angle-seeding and raised 
intraocular pressure. In this case, biopsy was not performed owing to the risk of haemorrhage. Overall, five patients showed raised intraocular pressure at presentation.

Median follow-up post-treatment was 40 months (range 6-65 months).

Tumour control was achieved in 14 patients (93\%). One patient showed local recurrence of melanoma requiring enucleation.

Five (33\%) patients developed symptomatic dry eye. Eight $(53 \%)$ patients developed glaucoma including the five patients noted pretreatment. In four patients, the intraocular pressure was controlled with topical medications only; two required repeated cyclodiode, one cyclocryotherapy and subsequent enucleation for phthisis, and one patient required enucleation for rubeosis.

Overall globe retention was achieved in $80 \%$ (12 out of 15 patients).

No patients developed metastases over the period of follow-up.

\section{Discussion}

Treatment options for iris melanoma depend on, among other things, the extent of the tumour, health of the eye and its fellow, and the age and general health of the patient. For the majority of patients, the natural history of iris melanomas is one of slow growth and low metastastic potential. The risk of metastases in focal melanomas has been reported to be as low as 3\%, ${ }^{1}$ whereas for diffuse lesions the rate is greater at approximately $13 \% .^{5}$ For this reason, the mainstay of treatment of focal lesions is local resection by iridectomy or iridocylcectomy, if the angle is involved. Such operations carry relatively low morbidity and a good visual prognosis. ${ }^{6}$

Treatment of diffuse melanomas or those with raised intraocular pressure is more difficult. Such lesions tend to behave more aggressively, and Shields et $a l^{7}$ have reported that up to $60 \%$ of cases with raised intraocular pressure may require enucleation. The same authors have reported success with brachytherapy utilising custom-made plaques with a variety of isotopes. ${ }^{8}$ That study showed a local control rate of $92 \%$ and an eye retention rate of $87 \%$ at 5 years. Their main complications were cataract $(70 \%)$ and glaucoma $(37 \%)$.

Although proton beam has been used extensively for posterior melanoma, there is little published data on its use for iris melanoma. ${ }^{3,4}$ In 2001, at the International Congress of Ocular Oncology in Amsterdam, Damato presented his experience of the use of proton beam with iris melanoma over a period of 5 years. ${ }^{9}$ Although follow-up was relatively short, the initial results with regard to recurrence rates and local complications were encouraging.

Our series included four cases of focal melanoma: one patient was a high myope with no perception of light in his fellow eye secondary to retinal detachment (Figure 1b, c and d), the other showed a highly vascular lesion with angle involvement and raised intraocular pressure, and two patients elected to undergo proton beam therapy rather than surgery. The remaining 11 patients showed diffuse (Figure 1e and f) or extensive nonresectable tumours, cases with raised intraocular pressure and included two cases of recurrence following previous local resection. Therefore, they represent a group who might be expected to fare poorly.

Overall, local control of tumour was achieved in $93 \%$ (one local recurrence). Despite this, enucleation was necessary in three cases (20\%).

In the remaining 12 patients, five (30\% of total) showed improved or stable visual acuity and seven suffered a loss of acuity ( $46 \%$ of total). Overall, eight patients had acuities better than $6 / 9$, one had $6 / 12$ and three patients had only hand movements. The cause of poor vision in these three individuals was glaucoma in one, glaucoma and cataract in another, and preexisting surgical aphakia in the third.

Cataracts developed in three patients postirradiation. One patient refused surgery and two underwent phacoemulsification although these eyes were subsequently enucleated for phthisis and rubeosis. Symptomatic dry eye developed in five cases $(36 \%)$ but was readily controlled with artificial tears. There were no cases of corneal melt, prolonged uveitis, radiation retinopathy, or papillopathy. No patient has developed systemic metastases to date.

The most frequent complication was of raised intraocular pressure, which was noted at presentation (and persisted) in five individuals and developed in a further three patients, making eight in total (53\%). In seven cases, the glaucoma was of open-angle type and in one case secondary to rubeosis. At presentation the mechanism for the raised pressure appeared to be outflow obstruction by melanoma. However, the fact that the intraocular pressure remained raised and that none of our patients showed a reduction of pressure postirradiation despite regression of the melanoma suggests that trabecular scarring may have played a role.

In summary, proton beam therapy proved effective in the management of cases of iris melanoma where local resection was either impossible or refused. Our local control and eye retention rates compare favourably to those achieved with brachytherapy. Overall, anterior segment seems to tolerate proton beam therapy well. The major complication is glaucoma, the management of which may be problematic. 


\section{References}

1 Geisse LJ, Robertson DM. Iris melanomas. Am J Ophthalmol 1985; 99: 634-648.

2 Jakobiec FA, Silbert G. Are most iris 'melanomas' really nevi? A clinicopathological study of 189 cases. Arch Ophthalmol 1981; 99(12): 2117-2132.

3 Zografos L. In: Tumeurs Intraoculaires. Societe Francaise d'Ophtalmologie et Masson: Paris, 2002 pp 299-302.

4 Munzenrider JE. Particle treatment of the eye. In: Sagerman RH, Alberti WE (eds). Radiotherapy of Intraocular and Orbital Tumors. Springer: Berlin, 2003, chapter 7, pp 59-60

5 Demirci H, Shields CL, Shields JA, Eagle RC, Honavar SG. Diffuse iris melanoma. Ophthalmology 2002; 109: 1553-1560.
6 Conway RM, Chua WC-T, Qureshi C, Billson FA. Primary iris melanomas: diagnostic features and outcome of conservative surgical treatment. Br J Ophthalmol 2001; 85: 848-854.

7 Shields CL, Materin MA, Shields JA, Gershenbaum E, Singh $\mathrm{AD}$, Smith A. Factors associated with elevated intraocular pressure in eyes with iris melanoma. Br J Ophthalmol 2001; 85: 666-669.

8 Shields CL, Naseripour M, Shields JA, Freire J, Cater J. Custom-designed plaque radiotherapy for nonresectable iris melanoma in 38 patients: tumor control and ocular complications. Am J Ophthalmol 2003; 135: 648-656.

9 Damato B, Kacperek A, Sheen M, Errington RD. Proton beam radiotherapy of iris melanoma. Presented at $\mathrm{Xth}$ International Congress of Ocular Oncology, Amsterdam, 2001. 